\title{
RBMXL1 is differentially expressed in the brains of patients with psychotic disorders.
}

Shahan Mamoor ${ }^{1}$

1'shahanmamoor@gmail.com

East Islip, NY, USA

We used public and published microarray data ${ }^{1,2}$ to identify the most significant gene expression changes in the brains of patients with psychotic disorders. We identified RBMXL1 as differentially expressed in the dorsolateral prefrontal cortex of patients with schizophrenia and schizoaffective disorder. In neurons of the dorsolateral prefrontal cortex, RBMXL1 was expressed at significantly lower levels in patients with psychotic disorders.

Keywords: schizophrenia, psychotic disorders, systems biology of schizophrenia, psychosis, RBMXL1.

PAGE 1 OF 12 
Psychotic disorders including schizophrenia and schizoaffective disorder are characterized by the presence of psychosis, or an inability to engage in reality testing 3,4 . Patients with psychotic disorders can experience positive as well as negative symptoms ${ }^{5}$. Positive symptoms include hallucinations, visual and auditory, as well as delusions, or fixed false beliefs ${ }^{5}$ Negative symptoms include anhedonia, or an inability to experience pleasure, as well as avolition, or loss of motivation 5 . To understand how specific regions of the brain differ most significantly in terms of gene expression in patients with psychotic disorders, we used published and public microarray data to compare global gene expression profiles of brain tissues from patients with psychotic disorders with that of non-affected, control subjects ${ }^{1,2}$. We found that RBMXL1 was among the genes whose expression changed most significantly in patients with schizophrenia in the dorsolateral prefrontal cortex, the layer 3 parvalbumin-positive neurons of the dorsolateral prefrontal cortex in patients with schizophrenia and in the layer 3 and layer 5 pyramidal neurons of the DLPFC in patients with schizophrenia. RBMXL1 may be relevant for future study of genes pertinent to the biology of psychotic disorders.

\section{Methods}

We used microarray datasets GSE93577 (1) and GSE87610 (2) for this differential gene expression analysis in conjunction with GEO2R. GSE93577 was generated using Affymetrix Human Genome U219 Array technology with $n=68$ for control subjects and $n=70$ for patients with schizophrenia and schizoaffective disorders, using layer 3 (L3) parvalbumin neurons of the dorsolateral prefrontal cortex (DLPFC). GSE87610 was generated using Affymetrix Human 
Genome U219 with $n=72$ for control subjects and $n=65$ for patients with schizophrenia, using L3 and L5 pyramidal neurons from the dorsolateral prefrontal cortex. The Benjamini and Hochberg method of p-value adjustment was used for ranking of differential expression but raw p-values were used for assessment of statistical significance of global differential expression. Logtransformation of data was auto-detected, and the NCBI generated category of platform annotation was used. A statistical test was performed to evaluate whether RBMXL1 expression was significantly different when comparing neurons from the DLPFC from control subjects and or from patients with schizophrenia or schizophrenia and schizoaffective disorder, respectively, using a two-tailed, unpaired t-test with Welch's correction. Only p-values less than 0.05 were considered statistically significant. We used PRISM for all statistical analyses (Version 8.4.0) $(455)$.

\section{Results}

We mined published and public microarray datasets to identify differentially expressed genes in the brains of patients with psychotic disorders.

RBMXL1 is differentially expressed in the PV+ L3 neurons dorsolateral prefrontal cortex of patients with schizophrenia and schizoaffective disorder.

We identified RBMXL1 as among the genes whose expression changed most significantly genome-wide when comparing the transcriptional profiles of parvalbumin-positive L3 neurons of the dorsolateral pre-frontal cortex (DLPFC) from control, non-affected subjects and from patients with schizophrenia or schizoaffective disorder (Table 1). When sorting each

PAGE 3 OF 12 
of the transcripts identified by microarray based on degree of change in expression between control subjects and patients with psychotic disorders, RBMXL1 ranked 147 out of 49386 total transcripts (Table 1). Differential expression of RBMXL1 in the PV+ L3 neurons of the DLPFC of patients with psychotic disorders in this dataset was statistically significant (Table 1; $p=9.23 \mathrm{E}-05)$.

RBMXL1 is differentially expressed in the L3 and L5 pyramidal neurons of the dorsolateral prefrontal cortex in patients with schizophrenia.

We mined a second microarray dataset to determine if differential expression of RBMXL1 in patients with psychotic disorders could be observed. We found here that RBMXL1 was among the genes most differentially expressed when comparing L3 and L5 pyramidal neurons from patients with schizophrenia to that of normal subjects. When sorting each of the transcripts identified by microarray based on degree of change in expression between control subjects and patients with schizophrenia, RBMXL1 ranked 3240 out of 49386 total transcripts (Table 1). Differential expression of RBMXL1 in the DLPFC of patients with schizophrenia was statistically significant (Table 2; $\mathrm{p}=1.39 \mathrm{E}-02)$.

RBMXL1 is expression is significantly lower in the PV+ L3 neurons dorsolateral prefrontal cortex of patients with schizophrenia and schizoaffective disorder.

We obtained exact mRNA expression levels for the differentially expressed RBMXL1 transcript, from control subjects and from patients with schizophrenia, to directly compare RBMXL1 expression levels in the PV+ L3 neurons of the DLPFC. We also performed a 
statistical test to determine if this difference in RBMXL1 expression was statistically significant. RBMXL1 was expressed at lower levels in the PV+ L3 neurons of the DLPFC in patients with schizophrenia, and this difference was statistically significant (Figure $1 ; p=0.0001$ ). We calculated a mean fold change of $0.8910 \pm 0.1517$ in RBMXL1 expression when comparing substantia nigra from patients with schizophrenia to that of control subjects (Table 1).

Thus, we found that RBMXL1 was among the most differentially expressed genes in the brains of patients with psychotic disorders, both in the dorsolateral prefrontal cortex and in the parvalbumin-positive L3 neurons of the dorsolateral prefrontal cortex, and that RBMXL1 was expressed at significantly lower PV + L3 neurons of the DLPFC in patients with schizophrenia.

\section{Discussion}

Retrogenes are processed copies of other genes, generated by insertion of copies of messenger RNA into the genome ${ }^{6}$. RNA binding motif protein, X-linked-like-1 (RBMXL1) is a nuclear RNA binding protein and retrogene of the RNA-binding motif protein X-linked RBMX containing an RNA-binding motif $(\mathrm{RBM})^{7}$. RBMX, also known as heterogenous ribonucleoprotein $\mathrm{G}(\mathrm{hnRNP} \mathrm{G})$, and found on the $\mathrm{X}$ chromosome is a homolog of RBMY, an RBM protein found on the Y chromosome ${ }^{7}$. RBMX-like sequences, like RBMXL1, lack intronid sequences in their genomic organization, demonstrating that they arose from retrotransposition 7 . RBMX and RBMY are an example of an $\mathrm{X} / \mathrm{Y}$ gene pair, wherein the $\mathrm{Y}$ copy acquires a testisspecific function while the $\mathrm{X}$ copy is subject to $\mathrm{X}$ inactivation in females.

PAGE 5 OF 12 
RBMXL1 has $96 \%$ identity at the nucleotide level with RBMX and encodes a protein of 390 amino acids in length?

There is little information available about the specific function of RBMXL1, but information regarding RBMXL1 can be gleaned by study of its derivative gene, RBMX. RBMX is glycosylated and possesses an RBM at its amino-terminus and a carboxy-terminus rich in serine, arginine and glycine ${ }^{8} . \quad$ hnRNPG is a reader of the $\mathrm{N}^{6}$-methyladenosine modification through interactions with RNA via the RBM and RGG (arginine glycine glycine) motifs 8 hnRNP G can bind the carboxy-terminus of RNA polymerase II (RNAPII) once phosphorylated co-transcriptionally associating with RNAPII and modulating RNA splicing; $\mathrm{m}^{6} \mathrm{~A}$ sites nean splice sites can thus affect hnRNP G binding8. RBMX can function as a regulator of the DNA damage response, localizing to DNA lesions and promoting homologous recombination through mechanisms involving BRCA2 expression dependent on poly (ADP-ribose) polymerase-1 (PARP-1) ${ }^{9}$. RBMX can also influence chromosome morphogenesis through interaction with the cohesin regulator Wapl and with cohesin subunits Scc1 and Smc3; depletion of RMBX results in loss of cohesin from centromeres before anaphase ${ }^{10}$. In amphibian oocytes, a central nascent RNA targeting domain (NTD) and not the RBM is required for hnRNP G interaction with nascent transcripts ${ }^{11}$. One study implicated RBMX and RBMXL1 in maintenance of "sonication-resistant" heterochromatin marked by H3K9me3; RBMX had the second highest enrichment at H3K9me3-marked chromatin; knockdown of RBMX and RBMXL1 enhanced expression of liver genes in fibroblasts, demonstrating that RBMX and RBMXL1 maintenance

PAGE 6 OF 12 
of sonication-resistant heterochromatin could serve as an impediment to reprogramming ${ }^{12}$.

Rbmx is expressed in the branchial arches and primordium in the developing zebrafish brain and expression of forebrain and hindbrain markers otx 2 and krox20 are decreased in Rbmx-deficient zebrafish ${ }^{13}$. In Xenopus, RBMX is also required for patterning of the anterior neural plate, neural crest development and muscle segmentation ${ }^{14}$. RBMX is a candidate gene for a form of moderate intellectual disability known as Shashi X-linked intellectual disability accompanied by craniofacial abnormalities ${ }^{15}$. Thus, RBMX functions in alternative splicing, can interact with RNA polymerase II, can mediate homologous recombination during DNA damage response, has $96 \%$ identity with its retrogene RBMX and is required for development of the brain in zebrafish and for the development of the anterior neural plate in Xenopus.

We observed significant changes in gene expression of a poorly characterized gene RBXML1, in the dorsolateral prefrontal cortex of patients with psychotic disorders, in L3 PV+ neurons and in L3 and L5 pyramidal neurons. RBMXL1 may be of relevance to the biology of psychotic disorders.

PAGE 7 OF 12 


\section{References}

1. GSE93577. Enwright J.F., Huo Z, Arion D, Corradi J.P, Tseng G., and Lewis D.A University of Pittsburgh, Psychiatry and Neuroscience. Pittsburgh, PA. https:/ www.ncbi.nlm.nih.gov/geo/query/acc.cgi?acc=GSE93577

2. Arion, D., Huo, Z., Enwright, J.F., Corradi, J.P., Tseng, G. and Lewis, D.A., 2017. Transcriptome alterations in prefrontal pyramidal cells distinguish schizophrenia from bipolar and major depressive disorders. Biological psychiatry, 82(8), pp.594-600.

3. Cannon, T.D., 2015. How schizophrenia develops: cognitive and brain mechanisms underlying onset of psychosis. Trends in cognitive sciences, 19(12), pp.744-756.

4. Arciniegas, D.B., 2015. Psychosis. Continuum: Lifelong Learning in Neurology, 21(3 Behavioral Neurology and Neuropsychiatry), p.715.

5. Andreasen, N.C., Nopoulos, P., Schultz, S., Miller, D., Gupta, S., Swayze, V. and Flaum, M., 1994. Positive and negative symptoms of schizophrenia: past, present, and future. Acta Psychiatrica Scandinavica, 90, pp.51-59.

6. Kabza, M., Ciomborowska, J. and Makałowska, I., 2014. RetrogeneDB-a database of animal retrogenes. Molecular biology and evolution, 31(7), pp.1646-1648.

7. Lingenfelter, P.A., Delbridge, M.L., Thomas, S., Hoekstra, H.E., Mitchell, M.J., Graves, J.A.M. and Disteche, C.M., 2001. Expression and conservation of processed copies of the RBMX gene. Mammalian Genome, 12(7), pp.538-545.

8. Zhou, K.I., Shi, H., Lyu, R., Wylder, A.C., Matuszek, Ż., Pan, J.N., He, C., Parisien, M. and Pan, T., 2019. Regulation of co-transcriptional pre-mRNA splicing by m6A through the lowcomplexity protein hnRNPG. Molecular cell, 76(1), pp.70-81.

9. Adamson, B., Smogorzewska, A., Sigoillot, F.D., King, R.W. and Elledge, S.J., 2012. A genome-wide homologous recombination screen identifies the RNA-binding protein RBMX as a component of the DNA-damage response. Nature cell biology, 14(3), pp.318-328.

10. Matsunaga, S., Takata, H., Morimoto, A., Hayashihara, K., Higashi, T., Akatsuchi, K., Mizusawa, E., Yamakawa, M., Ashida, M., Matsunaga, T.M. and Azuma, T., 2012. RBMX: a regulator for maintenance and centromeric protection of sister chromatid cohesion. Cell reports, 1(4), pp.299-308.

PAGE 8 OF 12 
11. Kanhoush, R., Beenders, B., Perrin, C., Moreau, J., Bellini, M. and Penrad-Mobayed, M., 2010. Novel domains in the hnRNP G/RBMX protein with distinct roles in RNA binding and targeting nascent transcripts. Nucleus, 1(1), pp.109-122.

12. Becker, J.S., McCarthy, R.L., Sidoli, S., Donahue, G., Kaeding, K.E., He, Z., Lin, S., Garcia, B.A. and Zaret, K.S., 2017. Genomic and proteomic resolution of heterochromatin and its restriction of alternate fate genes. Molecular cell, 68(6), pp.1023-1037.

13. Tsend-Ayush, E., O'Sullivan, L.A., Grützner, F.S., Onnebo, S.M., Lewis, R.S., Delbridge, M.L., Marshall Graves, J.A. and Ward, A.C., 2005. RBMX gene is essential for brain development in zebrafish. Developmental Dynamics, 234(3), pp.682-688.

14. Dichmann, D.S., Fletcher, R.B. and Harland, R.M., 2008. Expression cloning in Xenopus identifies RNA-binding proteins as regulators of embryogenesis and Rbmx as necessary for neural and muscle development. Developmental dynamics: an official publication of the American Association of Anatomists, 237(7), pp.1755-1766.

15. Shashi, V., Xie, P., Schoch, K., Goldstein, D.B., Howard, T.D., Berry, M.N., Schwartz, C.E., Cronin, K., Sliwa, S., Allen, A. and Need, A.C., 2015. The RBMX gene as a candidate for the Shashi X-linked intellectual disability syndrome. Clinical genetics, 88(4), pp.386-390.

PAGE 9 OF 12 


\begin{tabular}{|c|c|c|c|c|c|c|}
\hline Rank & ID & $p$-value & $\mathbf{t}$ & B & FC & Gene \\
\hline 147 & 11726096_s_at & $9.23 \mathrm{E}-05$ & -4.03 & 1.228245 & $\begin{array}{l}0.8910 \pm \\
0.1517\end{array}$ & RBMXL1 \\
\hline
\end{tabular}

Table 1: RBMXL1 is differentially expressed in L3 parvalbumin neurons of the dorsolateral prefrontal cortex in patients with schizophrenia and schizoaffective disorder.

The rank of differential expression, the probe/transcript ID, the p-value with respect to global differential expression, $t$, a moderated $t$-statistic, $B$, the log-odds of differential expression between the two groups compared, the fold change of RBMXL1 in neurons of the DLPFC when compared to DLPFC neurons from control subjects, the gene and gene name are listed in this chart. 


\begin{tabular}{|r|l|l|l|l|l|}
\hline Rank & ID & p-value & t & B & Gene \\
\hline 3240 & 11758378_s_at & $1.39 E-02$ & -2.4912189 & -3.12743 & RBMXL1 \\
\hline
\end{tabular}

Table 2: RBMXL1 is differentially expressed in L3 and L5 pyramidal neurons of the dorsolateral prefrontal cortex in patients with schizophrenia.

The rank of differential expression, the probe/transcript ID, the p-value with respect to global differential expression, $t$, a moderated $t$-statistic, $B$, the log-odds of differential expression between the two groups compared, the fold change of RBMXL1 in neurons of the DLPFC when compared to DLPFC neurons from control subjects, the gene and gene name are listed in this chart. 


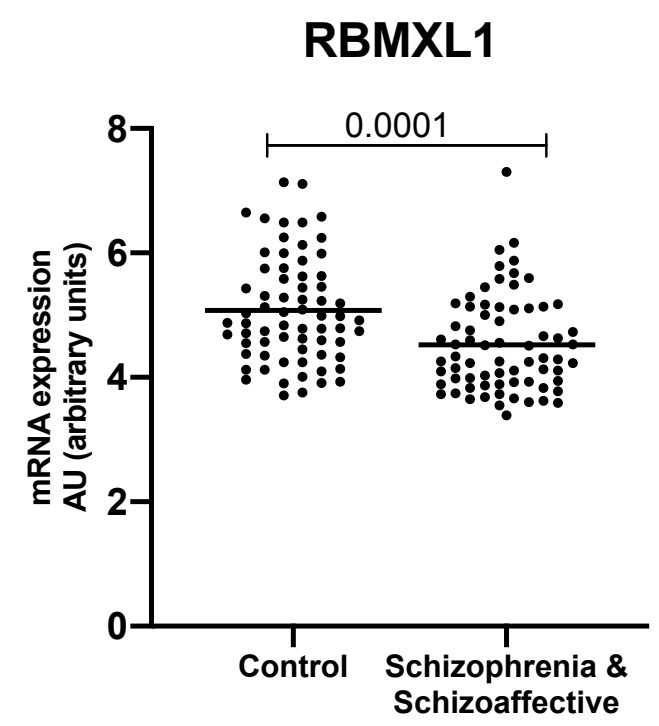

Figure 1: RBMXL1 is expressed at significantly lower levels in the L3 PV+ neurons of the DLPFC in patients with schizophrenia and schizoaffective disorder.

The mRNA expression of RBMXL1 in L3 PV+ DLPFC neurons from control subjects (left) and in the L3 PV+ DLPFC neurons from patients with schizophrenia or schizoaffective disorder (right) is represented with mean mRNA expression level marked and the result of a statistical test evaluating the significance of difference in RBMXL1 expression between unaffected L3 $\mathrm{PV}+$ neurons of the DLPFC and L3 PV+ neurons of the DLPFC from patients with psychotic disorders, a p-value, listed above. 